\title{
Modeling Separated Flows Using Stationary Discrete Vortices
}

\author{
Olga Aleksandrovna Averkova ${ }^{1}$, Ilya Valerievich Kryukov ${ }^{1}$, Kirill Victorovich Plotnikov ${ }^{1}$, Elena Igorevna \\ Tolmacheva $^{1}$ \& Ilya Vladimirovich Khodakov ${ }^{1}$ \\ ${ }^{1}$ Belgorod State Technological University named after V.G. Shoukhov, Kostyukova Street, 46, 308012, Belgorod, \\ Russia \\ Correspondence: Ilya Vladimirovich Khodakov, Belgorod State Technological University named after \\ V.G. Shoukhov, Kostyukova Street, 46, 308012, Belgorod, Russia. E-mail: mtrushin@mail.ru
}

Received: September 9, 2014

Accepted: September 30, $2014 \quad$ Online Published: February 28, 2015

doi:10.5539/mas.v9n3p291

URL: http://dx.doi.org/10.5539/mas.v9n3p291

\begin{abstract}
In many technological units (heating furnaces, kilns, aspiration hoods, etc.), a provision is made to include open ports through which undesirable air can flow. Air curtains have been widely applied to reduce the air flow rate by increasing the drag in these ports. However, this method of reducing the flow rate results in rather high operational cost due to unavoidable loss of electrical energy through air-curtain fans. Structural elements installed in front of the port inlet can provide an alternative. These structural elements ensure the drag increase without shutting off the effective cross section of the ports (in order to ensure implementation of technological operations of aggregate loading, displacement of different-type manipulators, or transport vehicles) and can be implemented with minimum cost. This study objective is to develop a method of mathematical modeling of separated flows upstream of suction slot openings within the SDVM (The discrete vortex method application is known in the stationary set-up framework). With use of discrete vortexes method in stationary statement, mathematical modeling method of detached flow on the input to the flat canals is constructed and discussed. The problem about detached flow on the input to the flat canal with screen is solved. Validity and adequacy of received results are demonstrated by the comparison with calculations, according to the method of N.E. Zukovsky and by experimental data. The developed method of mathematical modeling and computing algorithm of its realization may be used for detection of current available line, velocity field and form loss coefficient of input to the suction ducts, equipped by arbitrary system of thin screens.
\end{abstract}

Keywords: detached flows, discrete vortexes method, form loss coefficient

\section{Introduction}

The most exact data about flows in action spectrums of soaking-up openings are necessary for industrial ventilation tasks solving (Logachev, 2005-2014; Dorocenko, 2006).

There is ideal incompressible liquid analyzed (Logachev, 1995; Anzheurov, 2003). A vortex is separating from the sharp boundary edge and so-called free flow line is originating from the edge in the position unknown in advance. Traditionally this problem can be solved using the complex variable theory and ideal liquid jet theory methods (Averkova, Logachev, 2013). It is assumed that the velocity on the free flow line is constant. Using such methods ensures the most accurate results. There is an analytical solution obtained in some of the simplest cases. The problem often resolves oneself into quite lengthy non-linear equations with coefficients of unknown variables in the form of Cauchy type integrals. In the general case when a multiply connected flow region or, all the more, a three-dimensional space is considered solving the problems using the methods above is impossible.

The discrete vortex method application is known in the stationary set-up (SDVM) (Belotserkovsky1992; Lifanov, 2003; Logachev, 2006-2014) to solve problems of a separated flow of ideal incompressible liquid upstream of a round continuous tube (Logachev, 2004). The problem was solved in the axially symmetric set up with discrete vortex rings used as discrete vortices. A clearer boundary of the free streamline surface would be obtained by placing a free vortex ring on the sharp edge.

This study objective is to develop a method of mathematical modeling of separated flows upstream of suction slot openings within the SDVM framework. 


\section{Materials and Methods}

As an example, we considered a problem of a separated flow of ideal incompressible liquid upstream of a screened plane wall suction port (Figure 1) previously solved by various methods.

The following symbols were introduced: $N$ is the number of bound vortices of the upper boundary (above $O X$ axis); $N_{S}$ is the number of free vortices of the upper boundary. The vortex lying on sharp screen edge $C$ is considered to be a free one which follows from the theorem set forth in [15]: the bound vortex intensity (circulation) at the flow separation point is equal to zero. Bound vortices were also positioned at the boundary fracture points. There were reference points positioned between the bound vortices.

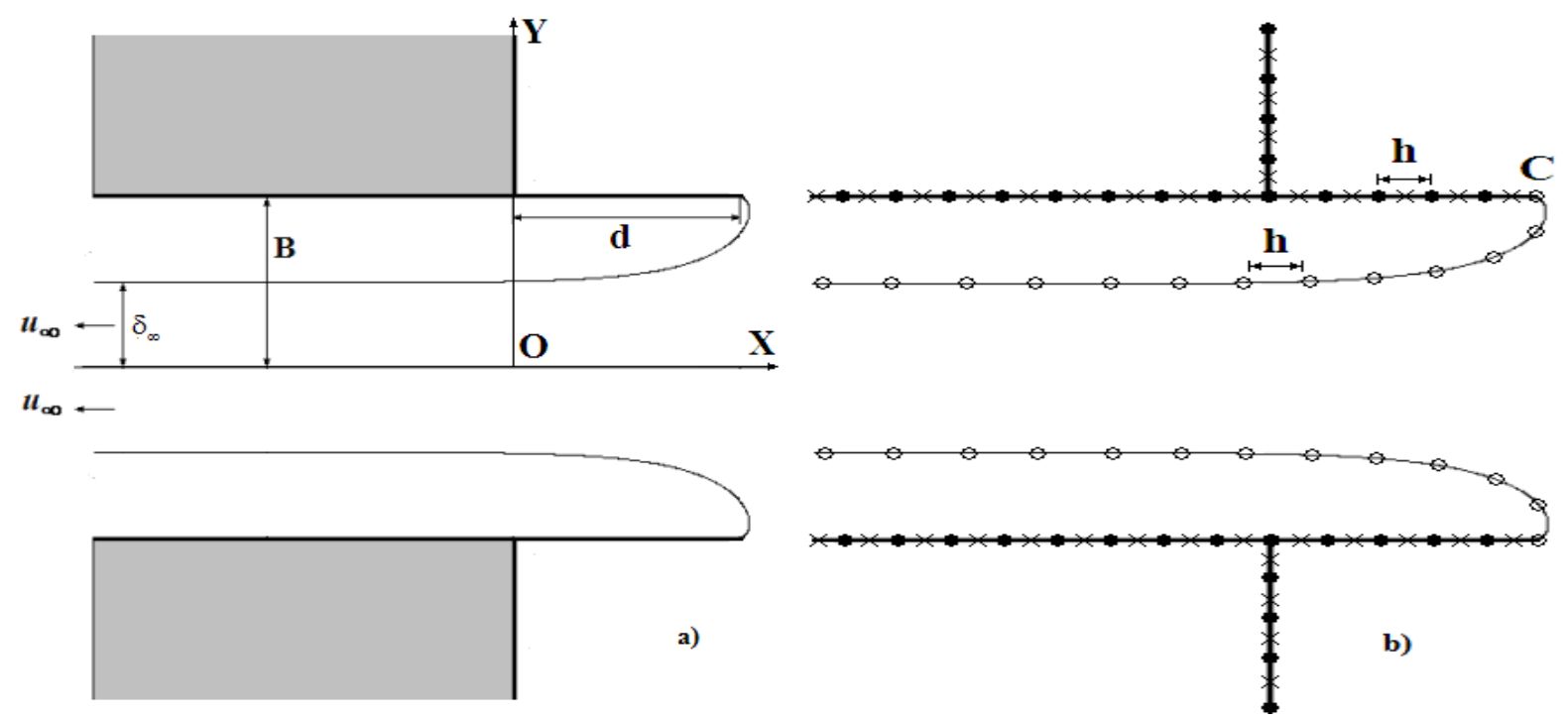

Figure 1. Regarding the problem set-up: a) physical flow area; b) range boundary discretization: ( * is for bound vortices, $\phi$ is for free vortices, $x$ is for reference points)

Point $\xi^{k}\left(\xi_{1}, \xi_{2}\right)$ is the bound vortex position point; $x^{p}\left(x_{1}, x_{2}\right)$ is the reference point. The action on point $x^{p}$ (velocity at this point) by the vortex of a single circulation located at point $\xi^{k}$ along the unit vector $\vec{n}=\left\{n_{1}, n_{2}\right\}$ is determined from the formula:

$$
G\left(x^{p}, \xi^{k}\right)=\frac{1}{2 \pi} \cdot \frac{\left(x_{1}-\xi_{1}\right) n_{2}-\left(x_{2}-\xi_{2}\right) n_{1}}{\left(x_{1}-\xi_{1}\right)^{2}+\left(x_{2}-\xi_{2}\right)^{2}}
$$

This vortex action on point $x^{p}$ is given by

$$
v_{n}\left(x^{p}\right)=G\left(x^{p}, \xi^{k}\right) T\left(\xi^{k}\right),
$$

where $T\left(\xi^{k}\right)$ is a circulation of the vortex located at point $\xi^{k} ; v_{n}\left(x^{p}\right)$ is the velocity at point $x^{p}$ along the unit vector $\vec{n}$.

With the boundary discretization method described above the number of bound vortices is equal to the number of reference points. Since the range boundary is symmetric around $O X$ axis and this axis is impermeable the circulation of symmetric vortices will be inverse:

$$
T\left(\xi^{k}\right)=-T\left(\xi^{k+N}\right), \text { where } k=\overline{1, N} .
$$

Fulfillment of this condition will automatically result in the non-circular flow condition (the sum of circulations of all vortices is equal to zero).

It was assumed that circulation of vortices on the required free flow line is constant and equal to [gamma]. The distance between free vortices is a constant value equal to $h$. The first approximation for the free flow line was chosen as follows. The first three vortices were position in parallel to $O Y$ starting from the sharp edge while the rest of the vortices were positioned in parallel to $O X$. 
The resultant action of all vortices on reference point $x^{p}$ along the outward normal is given by:

$$
\begin{gathered}
v_{n}\left(x^{p}\right)=\sum_{q=1}^{N} G\left(x^{p}, \xi^{q}\right) T\left(\xi^{q}\right)- \\
-\sum_{q=N+1}^{2 N} G\left(x^{p}, \xi^{q}\right) T\left(\xi^{q-N}\right)+\gamma \sum_{k=1}^{N_{S}} G\left(x^{p}, \zeta^{k}\right)- \\
-\gamma \sum_{k=N_{S+1}}^{2 N_{S}} G\left(x^{p}, \zeta^{k}\right)= \\
=\sum_{q=1}^{N}\left(G\left(x^{p}, \xi^{q}\right)-G\left(x^{p}, \xi^{q+N}\right)\right) T\left(\xi^{q}\right)+ \\
+\gamma \sum_{k=1}^{N_{S}}\left(G\left(x^{p}, \zeta^{k}\right)-G\left(x^{p}, \zeta^{k+N_{S}}\right)\right),
\end{gathered}
$$

where $\zeta^{k}$ is a free vortex position point.

Since $v_{n}\left(x^{p}\right)=0$ at all reference points, that is, the impermeability condition is fulfilled, expression (4) will be converted as follows:

$$
\begin{aligned}
& \sum_{q=1}^{N}\left(G\left(x^{p}, \xi^{q}\right)-G\left(x^{p}, \xi^{q+N}\right)\right) T\left(\xi^{q}\right)= \\
= & -\gamma \sum_{k=1}^{N_{S}}\left(G\left(x^{p}, \zeta^{k}\right)-G\left(x^{p}, \zeta^{k+N_{S}}\right)\right) .
\end{aligned}
$$

When $p$ is changed between 1 and $N$ expression (5) becomes a set of linear algebraic equations (SLAE) with $N$ unknown variables $T\left(\xi^{1}\right), T\left(\xi^{2}\right), \ldots, T\left(\xi^{N}\right)$. By solving this set using the Gaussian method with pivoting and determining unknown circulations of bound vortices we'll be able to compute the velocity at any point $x\left(x_{1}, x_{2}\right)$ of the range along any given direction from the formula:

$$
\begin{aligned}
v_{n}(x)= & \sum_{q=1}^{N}\left(G\left(x, \xi^{q}\right)-G\left(x, \xi^{q+N}\right)\right) T\left(\xi^{q}\right)+ \\
& +\gamma \sum_{k=1}^{N_{S}}\left(G\left(x, \zeta^{k}\right)-G\left(x, \zeta^{k+N_{S}}\right)\right) .
\end{aligned}
$$

If the distance from point $x$ to the vortex at point $\xi$ is less than $h / 2$ then $G(x, \xi)$ is given by:

$$
G(x, \xi)=\frac{r}{\pi h} \cdot \frac{\left(S_{1}-\xi_{1}\right) n_{2}-\left(S_{2}-\xi_{2}\right) n_{1}}{\left(S_{1}-\xi_{1}\right)^{2}+\left(S_{2}-\xi_{2}\right)^{2}},
$$

where $S_{1}=\frac{h}{2 r}\left(x_{1}-\xi_{1}\right)+\xi_{1} ; \quad S_{2}=\frac{h}{2 r}\left(x_{2}-\xi_{2}\right)+\xi_{2} ; r=\sqrt{\left(x_{1}-\xi_{1}\right)^{2}+\left(x_{2}-\xi_{2}\right)^{2}}$.

The second approximation for the free flow line was chosen as follows. There is a flow line plotted from sharp edge $C$ in the defined velocity field using the following formulas:

$$
x=x^{\prime}+v_{x} \Delta t, \quad y=y^{\prime}+v_{y} \Delta t,
$$

where $\Delta t$ is a quite small time increment (e.g. $\Delta t=0.0001) ;\left(x^{\prime}, y^{\prime}\right)$ is the previous position of the point on the flow line; $(x, y)$ is the previous point; $v_{x}, v_{y}$ are found from (6) at $\vec{n}=\{1 ; 0\}$ for $v_{x}$ and $\vec{n}=\{0 ; 1\}$ for $v_{y}$. As soon as the distance between point $(x, y)$ and the sharp edge becomes equal to $h$ a free vortex is placed at this point, i.e. it will be the second approximation for this free flow line point. Then, a flow line is plotted again using (8) until the distance between $(x, y)$ and the previous position of the free vortex becomes equal to $h$. A free vortex is placed at this point and so on.

After defining the second approximation for the free flow line there should be set of equations (5) resolved again and bound vortex circulations determined. Then, the third approximation is made for the free flow line using formulas (6)-(8) etc. This iteration process is repeated until the difference between the previous and next positions does not exceed the given accuracy. 
Since the range boundaries "break" at some distance from the suction port a situation occurs when the free flow line starts lifting up (for upper part of Figure1), i.e. its Y-coordinate starts increasing. That is why in this case $\mathrm{y}$-coordinate is fixed for all other points and is equal to the maximum decrease point Y-coordinate.

The word description of the computational algorithm built using design relations (1)-(8) is as follows.

1. There are matrixes of coordinates of reference points $k t[1 . . N, 1 . .2]$; coordinates of bound vortices $p v[1 . . N, 1 . .2]$; coordinates of free vortices $s v[1 . . N, 1 . .2]$ formed.

a. For the upper screen:

$$
\begin{aligned}
& k t[i, 1]=d-h\left(i-\frac{1}{2}\right) ; k t[i, 2]=B ; \\
& p v[i, 1]=k t[i, 1]-\frac{h}{2} ; p v[i, 2]=B ; \\
& i=\overline{1, N_{1}}, \text { where } k t\left[N_{1}, 1\right]=\frac{h}{2} ; p v\left[N_{1}, 1\right]=0 .
\end{aligned}
$$

b. For the upper vertical wall:

$$
\begin{aligned}
& k t[i, 1]=0 ; k t[i, 2]=B+h\left(k-\frac{1}{2}\right) ; \\
& p v[i, 1]=0 ; p v[i, 2]=k t[i, 2]+\frac{h}{2} ; \\
& i=\overline{N_{1}, N} ; \text { where } k t[N, 2]=k a l i b r-\frac{h}{2} ;
\end{aligned}
$$

kalibr is the vertical wall length (divisible by the suction port width $B$ );

$k=1,2, .$. until $\left(k t[N, 2]-k a l i b r+\frac{h}{2}\right)<0.000001$.

c. For the range boundary symmetric around $O X$ :

$$
\begin{aligned}
& k t[i, 1]=k t[i-n, 1] ; k t[i, 2]=-k t[i-n, 2] ; \\
& p v[i, 1]=p v[i-n, 1] ; p v[i, 2]=-p v[i-n, 2] ;
\end{aligned}
$$

where $i=\overline{N+1,2 N}$.

d. For the upper free flow line:

$$
\begin{aligned}
& s v[i, 1]=d ; s v[i, 2]=b-h(i-1) ; i=\overline{1,3} ; \\
& s v[i, 1]=d-h(i-3) ; s v[i, 2]=b-2 h ; i=\overline{4, k_{2}} ;
\end{aligned}
$$

where $k_{2}$ is an integral part of $\mathrm{kalibr} / \mathrm{h} ; N_{S}=i-1$.

e. For the lower free flow line:

$$
\begin{gathered}
s v[i, 1]=s v\left[i-N_{S}, 1\right] ; s v[i, 2] \\
i=\overline{N_{S}+1,2 N_{S}} .
\end{gathered}
$$

2. Forming a matrix of coefficients of unknown variables of SLAE (5):

$$
\begin{gathered}
\operatorname{kof}[p, q]=\operatorname{Skor}(k t[p], p v[q], e v n)- \\
-\operatorname{Skor}(k t[p], p v[q+n], e v n) ;
\end{gathered}
$$

where $p=\overline{1, N} ; q=\overline{1, N} ; \operatorname{env}[1 . .2]$ are outward normal coordinates:

$\operatorname{evn}[1]=0 ; \quad \operatorname{evn}[2]=-1 \quad$ at $k t[p, 2]=B ; \quad \operatorname{evn}[1]=0 ; \quad \operatorname{evn}[2]=1$ at $k t[p, 2]=-B ; \operatorname{evn}[1]=-1 ; \quad \operatorname{evn}[2]=0$ at $k t[p, 1]=0$;

$\operatorname{Skor}(k t[p], p v[q]$, evn $)$ is a velocity along evn vector direction at point $k t[p]$ induced by the single circulation vortex located at point $p v[q]$. It is calculated using formulas (1), (7). 
3. Forming a column of free terms for SLAE (5):

$$
\begin{gathered}
\operatorname{pr}[p]=-S v G \sum_{k=1}^{N_{S}}(\operatorname{Skor}(k t[p], s v[k], e v n)- \\
\left.-\operatorname{Skor}\left(k t[p], s v\left[k+N_{S}\right], \text { evn }\right)\right),
\end{gathered}
$$

where $p=\overline{1, N} ; S v G$ is a circulation of vortices on the free flow line.

4. Solving SLAE

$\sum_{q=1}^{N} k o f[p, q] \operatorname{circ}[q]=p r[p] ; p=\overline{1, N}$ using the Gaussian method with pivoting and determination of the matrix of circulations of bound vortices $\operatorname{circ}^{[1 . . N]}$ at the upper boundary. Accordingly, for the lower boundary: $\operatorname{circ}[i]=-\operatorname{circ}[i-N], \quad i=\overline{N+1,2 N}$.

5. Forming coordinates of the next approximation for the free flow line:

a. There is an initial point of the flow line given $x[1]=s v[1,1] ; x[2]=s v[1,2]$.

b. There is an increment in the flow velocity direction made, i.e. there are coordinates calculated:

$$
\begin{aligned}
& x[i]=x[i]+\operatorname{chag}\left(\sum_{q=1}^{2 N} \operatorname{circ}[q] \operatorname{Skor}(x, p v[q], \text { evn })+\right. \\
& +\operatorname{SvG} \sum_{q=1}^{N_{S}}(\operatorname{Skor}(x, \operatorname{sv}[q], \operatorname{evn})- \\
& \left.\left.-\operatorname{Skor}\left(x, \operatorname{sv}\left[q+N_{S}\right], \text { evn }\right)\right)\right),
\end{aligned}
$$

where $i=\overline{1,2} ; \operatorname{evn}[1]=1 ; \operatorname{evn}[2]=0$ at $i=1$ and $\operatorname{evn}[1]=0 ; \operatorname{evn}[2]=1$ at $i=2 ; \operatorname{chag}=0,0001$ is time increment.

c. As soon as the distance from point $x$ to the previous point of the free flow line is $\sqrt{(x[1]-s v[i-1,1])^{2}+(x[2]-s v[i-1,2])^{2}} \geq h$ there are coordinates of the next approximation determined for the free flow line coordinates $p s v[i]=x$.

d. If the flow line point Y-coordinate exceeds the previous point Y-coordinate proceed to step e) otherwise proceed to step b). The count interruption condition: $x[1]<s v\left[N_{S}, 1\right]$.

e. There are the free flow line points determined:

$p s v[i, 1]=p s v[i-1,1]-h ; \quad p s v[i, 2]=x[2]$.

The count interruption condition: $p s v[i, 1]<s v\left[N_{S}, 1\right]$.

f. Old coordinates of the free flow line are changed for new ones

$s v[i]=p s v[i] ; \quad s v\left[i+N_{S}, 1\right]=p v s[i, 1] ; \quad s v\left[i+N_{S}, 2\right]=-p s v[i, 2]$,

where $i=\overline{1, N_{S}}$.

6. Then proceed to step 3, i.e. determine the new approximation for the free flow line. This cycle is repeated as long as $\left|d b-p v\left[N_{S}, 2\right]\right|>0.0001$, where $d b=s v\left[N_{S}, 2\right]$ is Y-coordinate of the previous position of $N_{S}$ point of the free flow line.

After the free flow line boundary has been defined its coordinates and circulations of bound vortices will be written to a file. Then this data can be used to determine the field of velocities. The velocity at the given point $x(x[1], x[2])$ along the given direction evn $(\operatorname{evn}[1] ; \operatorname{evn}[2])$ will be determined from the formula: 


$$
\begin{aligned}
v=\sum_{q=1}^{2 N} \operatorname{circ}[q] \operatorname{Skor} & (x, p v[q], \text { evn })++\operatorname{SvG} \sum_{q=1}^{N_{S}}(\operatorname{Skor}(x, s v[q], \text { evn })- \\
- & \left.\operatorname{Skor}\left(x, s v\left[q+N_{S}\right], \text { evn }\right)\right) .
\end{aligned}
$$

\section{Results}

Using the developed software program there were dimensionless velocity profiles $\left(u_{x} / u_{\infty} ; u_{y} / u_{\infty}\right)$ and free flow line coordinates calculated. For convenience of comparison of calculations made with various methods they are represented in another system of coordinates (Figure 2).

Continuous lines on all figures denote calculations made using The Zukovsky method while dotted and dash-dotted lines demote calculations made using the SDVM. As seen, the velocity and free flow line coordinates actually coincide. The biggest difference for the horizontal velocity component is observed in section $x=0.8$ but it does not exceed $2 \%$ versus calculations made using the Zukovsky method. The discretization interval in calculations made using the DVM is $h=0.0125, u_{\infty}$ was assumed to be the velocity on the suction axis 2 gauges away from the suction port.

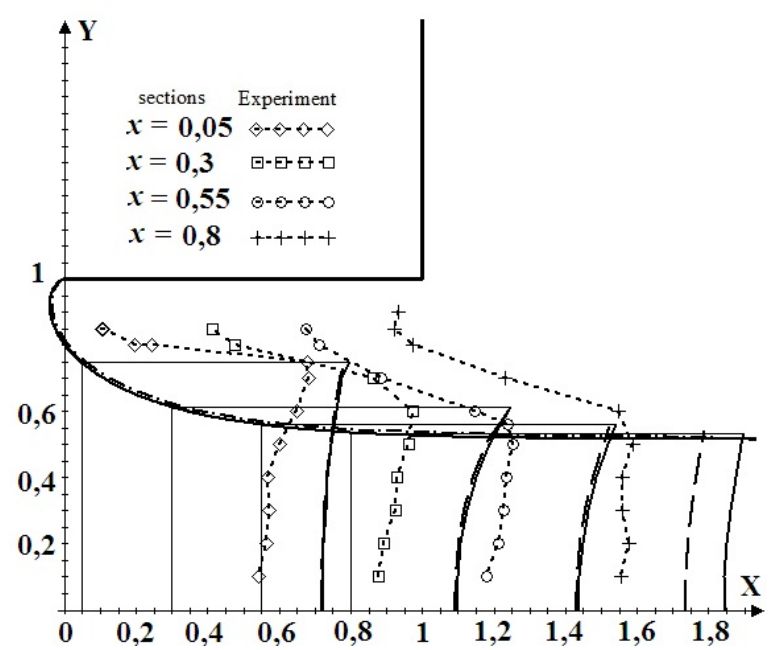

Figure 2. Free flow line and horizontal velocity component profiles

The vertical wall length and the distance of the last free flow line point from the suction port is equal to 8 gauges $(8 C)$.

The ratio of the difference of velocity $u_{\infty}$ and average slot velocity $u_{\infty} \delta_{\infty} / B$ to the average velocity is $\Delta u=B / \delta_{\infty}-1$ which was used as the local drag factor criterion in Chapter 4. Calculation of $\Delta u$ using the discrete vortex method demonstrates better conformance to the experimental data for the local drag factor $\zeta$ than in case with The Zukovsky method (Figure 3).

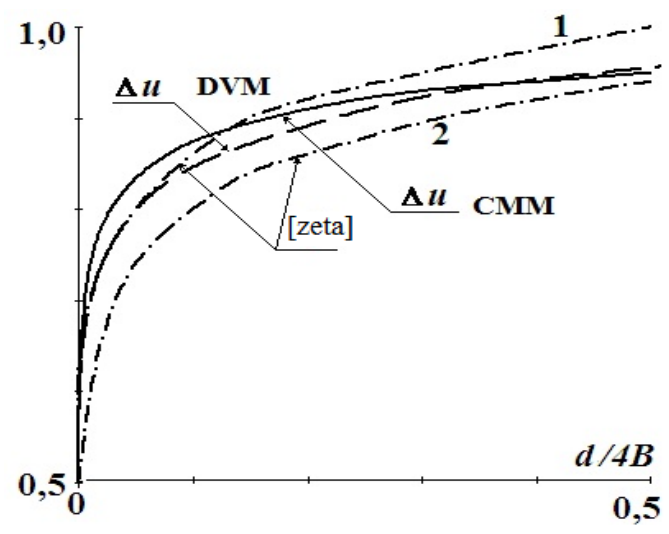

Figure 3. Variation in the local drag factor upstream of a flat pipe $(\zeta)$ and in the jet separation velocity $(\Delta u)$ with increase in the offset length $(d / 4 B)$ : dash-dotted lines denote experimental data by I.E. Idelchik, 1977 (curve 1 is for the relative tube wall thickness $\delta / D_{g}=0$; curve 2 is for $\delta / D_{g}=0.004$ ) 


\section{Discussion}

Applied problems of industrial aerodynamics most often focus on reducing the drag of a suction slot (Khanzhonokov, 1959; Nosova 1959; Idelchik, 1977). But increasing this drag is equally important. First of all, it relates to the practical reducing of the intake of the gaseous medium through openings when there are no options of sealing left. One of the technical means to reduce unwanted air inflows is to use special screens which increase the drag and reduce the flow coefficients of air entering through minimized openings. These tasks can occur, say, for specialists engaged in confinement of harmful emissions by local exhaust ventilation (Logachev, 2005), as well as in various heat and power plants.

The ideal fluid theory, in particular the method by N.E. Zhukovsky, was used in the study of separated flows at the inlet into flat channels (Logachev, 2010) due to the fact that it allows determining not only the kinematic characteristic of the flow, but also the deterministic boundary of the flow. For example, the jet thickness, which is used to estimate the air drag at the inlet into holes.

Using the discrete vortex method in the non-stationary set up (NDVM) (Belotserkovsky, Lifanov 1995; Logachev, 2005-2010) enables plotting free lines and streamline surfaces. However, according to numerical experiments for problems concerning suction spectra of exhaust slots, jet contraction $\delta_{\infty}$ at a considerable distance from the suction port remains virtually unchanged while some geometric dimensions are changing. In addition, this value pulsates in time and needs to be averaged over time. Therefore using the NDVM to analyze $\delta_{\infty}$, determine the boundaries of separated flows and the relation of these values with air drag of suction inlets presents a certain problem.

Novelty of this work is the development of the method of the detachable current on the entrance to the soaking-up channels with use of stationary discrete vortexes. This method allows to define the border of a detachable current and compression coefficient of the stream which is necessary for determination of the local resistance coefficient. Simulating experiment with use of the method of simulation of RANS (Reynolds Averaged Navier-Stokes) from the Fluent software package didn't allow to define such distinct border of the jet separation. The method developed on the basis of stationary discrete vortexes gives the results which correspond more to the natural experiment.

\section{Conclusion}

Thus, the mathematical model of separated flows elaborated based on the SDVM allows for obtaining an adequate flow pattern and its application will enable resolving a number of problems of separated flow air mechanics. Further development of this method will consist in the accounting of the thin closed bodies in a range of operation of exhaust channels. It will allow to operate a stream separation on an entrance to soaking-up channels. It is planned to consider axisymmetric tasks also. In particular to investigate influence of thin screens on coefficient of local resistance of an entrance to round soaking-up channels. It is necessary to specify interrelation of coefficient of local resistance and size of compression of a stream. For this purpose it is necessary to make natural experiment. And, at last, it represents great scientific interest to consider a stream separation from sharp edges of the soaking-up channel in full three-dimensional statement.

\section{Acknowledgement}

The researchers are being supported by the RFBR (project number 14-41-08005 r_ofi_m) and Belgorod region.

\section{References}

Anzheurov, N. M., Logachev, I. N., Alifanova, A. I., \& Logachev, K. I. (2005). Special aspects of calculation of aspiration volumes at powdery materials overloads with increased autohesion properties. Vestnik of Belgorod State Technological University named after V. G. Shukhov, 12, 9-13.

Averkova, O. A., Logachev, I. N., Logachev, K. I., \& Khodakov, I. V. (2014). Modeling detached flows at the inlet to round suction flues with annular screens. Refractories and Industrial Ceramics, 54(5), 425-429. http://dx.doi.org/10.1007/s11148-014-9625-Z

Averkova, O. A., Zorya, V. U., Logachev, I. N., \& Logachev, K. I. (2010). Numerical simulation of air currents at the inlet to slot leaks of ventilation covers. Refractories and Industrial Ceramics, 51(3), 177-182. http://dx.doi.org/10.1007/s11148-010-9284-7

Averkova, O. A., Logachev, I. N., Logachev, K. I., \& Logachev, A. K. (2013). The principles of separated flow at the inlet of the protruding duct with screens. TsAGI Science Journal, 2, 219-243. http://dx.doi.org/10.1615/TsAGISciJ.v44.i2.40

Averkova, O. A., Zorya, V. Y., \& Logachev, K. I. (2007). Behavior of aerosol particles in suction bunker of 
standard design. Chemical and Petroleum Engineering, 43(11), 686-690. http://dx.doi.org/10.1007/s10556-007-0123-8

Belotserkovsky, S. M., \& Lifanov, I. K. (1992). Method of Discrete Vortices. CRC Press, Boca Raton. ISBN: $9780849393075, \mathrm{pp}: 464$.

Belotserkovsky, S. M., Kotovskii, V. N., Nisht, M. I., \& Fedorov, R. M. (1992). Two-Dimensional Separated Flows. CRC Press, Boca Raton. ISBN: 9780849393068, pp: 320.

Doroshenko, Y. A., Zorya, V. Y., \& Logachev, K. I. (2008). Development of the ecological management system at the industrial enterprise. Vestnik of Belgorod State Technological University named after V.G. Shukhov, 3, 85-87.

Doroshenko, Y. A., Zorya, V. Y., \& Logachev, K. I. (2008). Legal bases of labor and environmental protection at dusty productions. Vestnik of Belgorod State Technological University named after V.G. Shukhov, 3, 59-60.

Idelchik, I. E. (1977). Hydraulic resistance guide book. M: Mashinostroeniye, pp: 559.

Khanzhonokov, V. N. (1959). Reduction in aerodynamic drag of openings using ring shaped ribs and grooves. Industrial Aerodynamics, 12, 181-196.

Lifanov, I. K., Poltavskii, L. N., \& Vainikko, M. M. (2003). Hypersingular Integral Equations and Their Applications. CRC Press, Boca Raton. ISBN: 9780415309981, pp: 408. http://dx.doi.org/10.1201/9780203402160

Logachev, I. N., \& Logachev, K. I. (2005). Aerodynamic basis of suctions. Khimizdat, S-Pb. ISBN:5-93808-051-9, pp: 659.

Logachev, I. N., \& Logachev, K. I. (2014). Industrial air quality and ventilation: controlling dust emissions. CRC Press, Boca Raton. ISBN:9781482222166, pp: 417. http://dx.doi.org/10.1201/b16549

Logachev, I. N., Logachev, K. I., \& Averkova, O. A. (2010). Mathematical modeling of separated flows at inlet of a shielded flat duct. Computational methods and programming, 11(1), 68-77.

Logachev, I. N., Logachev, K. I., \& Averkova, O. A. (2013). Methods and Means of Reducing the Power Requirements of Ventilation Systems in the Transfer of Free-Flowing Materials. Refractories and Industrial Ceramics, 54(3), 258-262. http://dx.doi.org/10.1007/s11148-013-9585-8

Logachev, I. N., Logachev, K. I., \& Neikov, O. D. (1995). Localization of dust generation during the pressing of powders. Powder Metallurgy and Metal Ceramics, 34(3-4), 203-206. http://dx.doi.org/10.1007/BF00559570

Logachev, I. N., Logachev, K. I., Zorya, V. Y., \& Averkova, O. A. (2010). Modeling of separated flows next to suction slots. Computational methods and programming, 11, 43-52.

Logachev, K. I., \& Anzheurov, N. M. (2003). Flow Analysis of Slit-Type Suction Ports Shielded with Slender Visors. Refractories and Industrial Ceramics, 44(3), 145-148. http://dx.doi.org/10.1023/A:1026396016208

Logachev, K. I., \& Posokhin, V. N. (2004). Calculation of a Flow in the Vicinity of a Round Suction Pipe. Izv. VUZ. Aviatsionnaya Tekhnika, 1, 29-32.

Logachev, K. I., Puzanok, A. I., \& Zorya, V. Y. (2006). Numerical study of aerosol dust behavior in aspiration bunker. ECCOMAS CFD 2006 - European Conference on Computational Fluid Dynamics, e-Book Full Papers, pp: 11.

Logachev, K. I., Puzanok, A. I., \& Selivanov, E. V. (2005). Experimental determination of local resistance coefficients of slot-like profiled ventilating suctions. Vestnik of Belgorod State Technological University named after V.G. Shukhov, 12, 38-45.

Nosova, M. M. (1959). Resistance of shielded inlet and outlet pipes. Industrial aerodynamics, 12, 197-215.

\section{Copyrights}

Copyright for this article is retained by the author(s), with first publication rights granted to the journal.

This is an open-access article distributed under the terms and conditions of the Creative Commons Attribution license (http://creativecommons.org/licenses/by/3.0/). 